\title{
The 15th International Biophilia Rehabilitation Conference Greetings from BRA in Japan
}

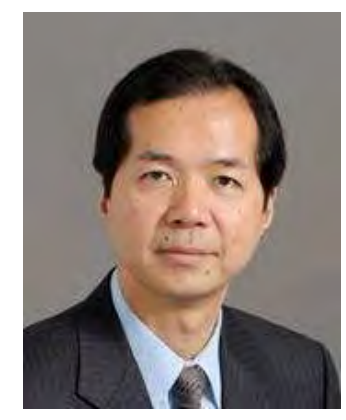

\author{
Prof. Toshiyuki Tanaka \\ Department of Applied Physics and Physico-Informatics \\ Faculty of Science and Technology, Keio University, Japan
}

It is a great pleasure to hold the 14th International Biophilia Rehabilitation Conference (15th IBRC) in Chieti-Pescara, Italy, on June 28-29, 2018. I am Toshiyuki Tanaka, chairperson of Biophilia Rehabilitation Academy in Japan. I am part of the Faculty of Science and Technology at Keio University, which is a longestablished private institution founded in Japan in 1858 by Yukichi Fukuzawa. Keio University is well known to researchers worldwide for the students and professors who produce high-quality research.

My research field is pattern measurement, including medical image processing and computer-aided diagnosis. The number of patients has increased in many countries, in step with the aging of the population. Medical devices such as fMRI, fNIRS, and X-ray CT become widely used to support the work of doctors. Although the precision of diagnosis has risen markedly, it seems that the burdens on doctors have not lessened. To reduce these burdens, we are developing a diagnosis support system using medical image processing.

We have promoted the construction of a new rehabilitation system that one physical therapist treats several patients with easy devices, which was called "Takizawa method". The international Biophilia Rehabilitation Academy (IBRA), which is a sisterhood institute of BRA in Japan, has worldwide revolved the promotion of new rehabilitation system. In consequence, IBRA has achieved a harmonious relationship with Poland, Italy, Romania, and so on. In this year, we have an international conference in cooperation with Chieti University again, where the 10th IBRC was held in 2013. We appreciate the chair of the organizing committee in ChietiPescara. The BRA in Japan shall be glad to serve IBRC 2018 in any way in our power.

Through a workshop and research exchange, we hope that the 15th IBRC will provide the opportunity to introduce important innovations in the field of the rehabilitation. 